Revue scientifique sur la conception et l'aménagement de l'espace

$16 \mid 2017$

Arbres et paysages

\title{
L'arbre urbain à Tunis : entre fonctionnalités et croyances
}

The Human Tree in Tunis: Functions and Beliefs

Myriam Bennour Azooz

\section{OpenEdition}

\section{Journals}

Édition électronique

URL : http://journals.openedition.org/paysage/4939

DOI : 10.4000/paysage.4939

ISSN : 1969-6124

\section{Éditeur :}

École nationale supérieure du paysage de Versailles-Marseille, Institut national des sciences appliquées Centre Val de Loire - École de la nature et du paysage, École nationale supérieure d'architecture et de paysage de Bordeaux, École nationale supérieure d'architecture et de paysage de Lille, Agrocampus Angers

\section{Référence électronique}

Myriam Bennour Azooz, "L'arbre urbain à Tunis : entre fonctionnalités et croyances », Projets de paysage [En ligne], 16 | 2017, mis en ligne le 09 juillet 2017, consulté le 30 avril 2020. URL : http:// journals.openedition.org/paysage/4939; DOI : https://doi.org/10.4000/paysage.4939

Ce document a été généré automatiquement le 30 avril 2020.

Projets de paysage 


\section{L'arbre urbain à Tunis : entre fonctionnalités et croyances}

The Human Tree in Tunis: Functions and Beliefs

Myriam Bennour Azooz

1 L'arbre urbain ne se réduit pas à une simple présence physique et fonctionnelle dans l'espace de la ville. Il rend des services écosystémiques importants, notamment de régulation environnementale et sociale du milieu urbain. Il revêt également, pour bon nombre de citadins, une signification symbolique forte. C'est le cas en Tunisie et notamment à Tunis où a été réalisée une thèse de doctorat consacrée à la patrimonialisation des arbres urbains (Bennour, 2014).

2 S'agissant des arbres, il y a un apparent paradoxe à parler de patrimoine, car ce dernier est ce à quoi on s'identifie et que l'on souhaite conserver en l'état ou amélioré, alors que ce qui est vivant est appelé à mourir et à disparaître. Le Dictionnaire de la géographie et de l'espace des sociétés définit, à ce propos, le patrimoine comme étant « un ensemble d'attributs, de représentations et de pratiques fixés sur un objet non contemporain dont est décrétée collectivement l'importance, et qui exige qu'on le conserve et qu'on le transmette. » (Levy et Lussault, 2003). Ce qui ressort tout d'abord de cette définition, c'est que le patrimoine est une notion étroitement liée à l'histoire humaine. Plus encore, il est le support d'une mémoire collective. On peut retenir aussi que cette notion est tout aussi liée à celle de transmission, condition essentielle lorsque l'on parle de patrimoine.

3 Dans la langue arabe, le terme tourâtes (patrimoine) est dérivé du verbe waratha qui signifie hériter. Il reste néanmoins distinct de la notion d'héritage qui se dit irth en arabe. Alors que dans la culture occidentale, «la notion (courante) de patrimoine [...] reste encore très largement attachée à la mort et aux héritages qui en résultent et se présente donc avec tous les atours du sacré et du tabou » (ibid.). Dans la culture arabe, elle est tributaire de celle de transmission à laquelle elle est intimement liée. On peut lire dans les dictionnaires anciens que le patrimoine est « ce qui se transmet en termes de traditions, de sciences, de littérature, d'arts, et ce que produisent les générations passées comme idéologies, poésies, chants ou danses, d'une génération à une autre » 
(Lissan al-Arab, 1879-1888). Ici aussi, on remarque que l'objectif premier du patrimoine est d'être transmis. On peut relever également dans cette définition - une des premières émises sur la notion de patrimoine dans la culture arabe - que ce que l'on qualifie de patrimoine digne d'être transmis relève plus de ce qui est aujourd'hui communément appelé patrimoine culturel immatériel en Occident. En effet, on y évoque essentiellement les traditions, la littérature dont la poésie et les chants, et même les idéologies, tous étant distinctifs d'une culture donnée et porteurs de signes identitaires très forts.

4 Une prise de conscience collective du patrimoine, à différents niveaux et à des époques successives, a fait passer dans la culture occidentale le patrimoine de la famille à la nation (Babelon et Chastel, 1994). En présence des risques de disparition du fait du temps et de l'évolution de la vie sociale et économique, une nouvelle prise de conscience a fait naître dans la communauté internationale à partir de 1972 un sens patrimonial encore plus élargi. Elle a fait du patrimoine l'héritage de toute l'humanité en considérant que "la valeur universelle exceptionnelle signifie une importance culturelle et/ou naturelle telle qu'elle transcende les frontières nationales et qu'elle représente le même caractère inestimable pour les générations actuelles et futures de l'ensemble de l'humanité » (déclaration de Stockholm, conférence des Nations unies sur l'environnement, 1972).

Qu'en est-il des arbres? Aujourd'hui, la notion de patrimoine vivant évolutif existe puisque des sites sont classés au patrimoine mondial de l'Unesco comme paysage culturel évolutif. Cette notion de patrimoine vivant évolutif admet que les caractéristiques patrimoniales elles-mêmes peuvent évoluer, voire même être remplacées par d'autres, tant que la qualité patrimoniale demeure de même importance. Comme l'explique le paysagiste Pierre-Marie Tricaud dans sa thèse de doctorat (2010), l'évolution qui transforme un être vivant, un objet ou un milieu n'est pas contradictoire avec sa valeur patrimoniale de transmission. Elle peut même, dans certains cas, augmenter cette valeur en améliorant ce qui a été hérité. La constance, dans le patrimoine, devient donc dépendante du regard de ceux qui en définissent la valeur patrimoniale. À charge, pour ceux qui ont patrimonialisé cet héritage, d'assurer sa continuité dans le temps par sa reproduction in situ ou non. C'est ce que font les réserves naturelles et les parcs nationaux. C'est aussi ce que font les jardiniers quand ils replantent un alignement de Ficus nitida ou de Washingtonia filifera dans les avenues de Tunis. Dans les deux cas il y a transmission d'un patrimoine naturel vivant public aux générations futures.

6 Concernant l'arbre urbain de Tunis, trois hypothèses peuvent être explorées. La première est liée au rôle environnemental et esthétique des arbres. La deuxième hypothèse soutient que les arbres plantés dans les villes peuvent être considérés comme des monuments au sens où ils évoquent la mémoire d'un temps passé, soit parce qu'ils sont très vieux et méritent le respect et le soin, soit parce qu'ils ont accompagné des événements urbains publics dont il convient de se souvenir. La dernière hypothèse relève de l'attachement intime, souvent symbolique, que les habitants peuvent accorder à tel ou tel arbre, public ou privé, isolé ou en tant que structure végétale (alignement par exemple). Mais selon que les uns sont sensibles à l'histoire urbaine ou à leurs souvenirs personnels, et les autres à des engagements militants pour la qualité de vie en ville, les arbres désignés varieront et parfois aucun ne sera cité. Si ces hypothèses sont plausibles, il faut aussi admettre qu'il existe des obstacles à considérer 
l'arbre comme patrimoine. Parce que vivant, l'arbre est périssable et ne peut être conservé comme un monument architectural. Et parce qu'en tant que décor, il est susceptible d'évoluer selon les goûts des sociétés et leurs cultures. Le muséifier serait alors discutable.

7 Pour tenter d'éclairer ce sujet, l'étude s'est appuyée sur des enquêtes semi-directives auprès d'un échantillon de citadins. Une centaine de personnes ont été interrogées sur les trois secteurs de la zone d'étude : la médina, des quartiers modestes à l'ouest et au nord-ouest, et des quartiers aisés au nord. L'objectif était de faire ressortir leurs représentations des arbres urbains. S'y ajoutent quinze professionnels de l'arbre dans le domaine public: des fonctionnaires de la municipalité de Tunis et du service des Espaces verts, ainsi que des cadres de l'Agence de protection de l'environnement. L'objectif était de cerner la place accordée à l'arbre par les pouvoirs publics. L'enquête a suggéré qu'un processus de patrimonialisation des arbres urbains est d'ores et déjà en cours. Ce processus est spontané, c'est-à-dire qu'il ne résulte pas nécessairement d'une protection institutionnelle, comme cela a été observé dans certains pays, notamment en France (Tollis, 2013). Il puise sa source dans l'imaginaire des Tunisois.

8 En effet, le rôle utilitaire des arbres ne doit pas masquer les valeurs historiques, patrimoniales et politiques attribuées aux différentes espèces. Ainsi, bien qu'il existe aujourd'hui une forte relation affective liée à la perception sensible, principalement à la vue (port, intégration paysagère) mais aussi à l'odeur (parfum), l'esthétique ne suffit pas à rendre compte des multiples relations possibles à une espèce arborée (Bennour, 2012). Les conclusions de cette thèse (Bennour, 2014) soulèvent différentes interrogations, d'autant plus vives qu'elles questionnent les pratiques actuelles de gestion publique et privative. Le développement des politiques publiques en faveur de l'arbre et du végétal implique que les acteurs concernés puissent disposer d'outils permettant de connaître le contexte dans lequel ils vont être amenés à prendre des décisions. Il paraît en effet indispensable que les gestionnaires disposent d'un état des lieux clair du rôle de l'arbre, aussi bien dans l'espace matériel de la ville que dans l'esprit des Tunisois.

9 Sur quels critères faut-il alors se baser pour choisir de conserver un arbre urbain en tant que patrimoine à transmettre pour les Tunisois? Les résultats de la thèse nous amènent à envisager trois caractères significatifs de l'arbre à Tunis (décor urbain, porteur de mémoire et symbole spirituel) pour éclairer les choix de plantation, de protection et de transmission.

\section{L'arbre comme décor urbain}

10 L'arbre urbain à Tunis est investi d'une fonction esthétique majeure par les citadins. Les frondaisons des arbres urbains offrent en effet une palette infinie de perceptions agréables. Car c'est un fait indéniable que l'arbre est devenu depuis la période coloniale un élément constitutif majeur des paysages urbains. Échelle intermédiaire par rapport au bâti de grande hauteur, l'arbre contribue, par sa présence, à la perception d'une ville à taille humaine. En plus d'articuler les espaces publics entre eux et avec les éléments architecturaux et minéraux voisins, les arbres permettent également la création d'ambiances diversifiées et changeantes tout en structurant le paysage.

11 À Tunis, l'arbre se présente à différentes échelles: celle de la voirie sous forme d'alignements et celle des parcs, en isolé ou en bosquet, sont les plus répandues en 
ville. Notons que ces formes, apportées par les colons, n'ont pour ainsi dire « quasiment pas évolué depuis la période du Paris haussmannien » (responsable de la municipalité de Tunis, 2013) comme on peut le constater dans la figure 1, et qui a servi de modèle (André, 1879).

Figure 1. Photographies, de la période coloniale et d'aujourd'hui, de l'avenue de Carthage au centreville et de la place de la Résidence sur l'avenue Bourguiba, exemples de la pérennité des plantations coloniales

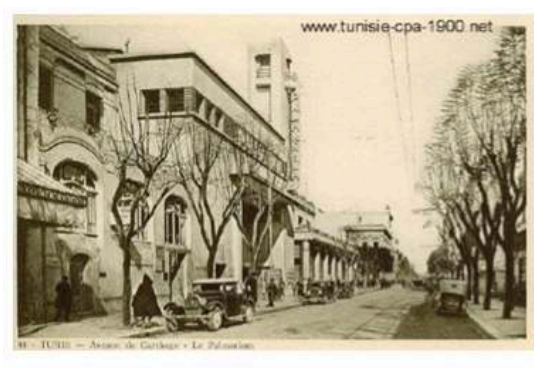

Figure 1a: Carte postale ancienne de l'avenue de Carthage plantée de Jacaranda mimoesifolia, vers 1930.

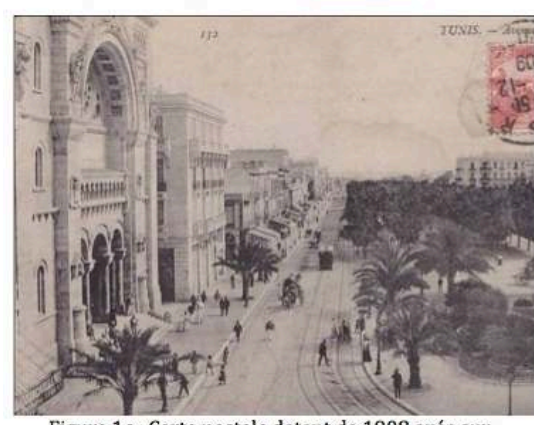

Figure 1c : Carte postale datant de 1908 axée sur l'avenue Jules-Ferry, montrant la place de la Résidence faisant face à la grande cathédrale plantée de Washingtonia filifera et de Phoenix dactylifera. Collection privée.

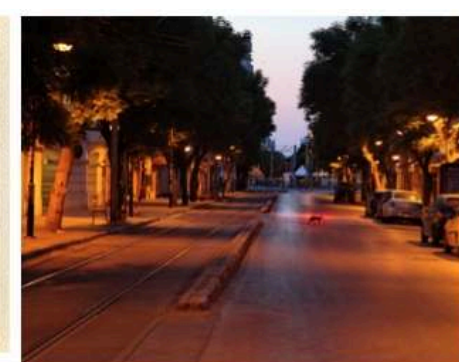

Figure $1 \mathrm{~b}$ : Avenue de Carthage aujourd'hui, avec son alignement de Jacaranda mimcesifolia.

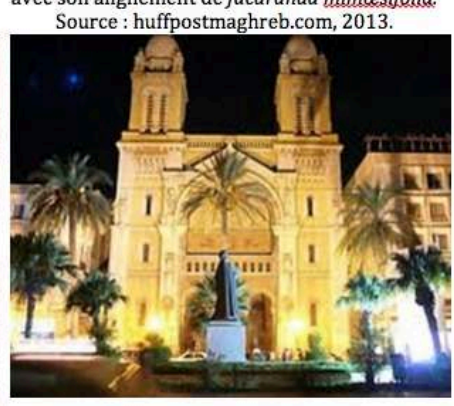

Figure 1d : Place en face de la cathédrale SaintVincent-de-Paul de Tunis sur l'avenue HabibBourguiba. La vue est cadrée par des Washingtonia filifera et de Phoenix dactylifera. Source : htunisie360, 2014.

Le choix a été fait de chercher certaines photographies sur Internet du fait de l'interdiction en Tunisie de prendre des photos de zones jugées sensibles.

\section{L'échelle de la voirie : l'alignement}

La ville européenne de Tunis est faite de rues, d'avenues, de places, de chemins et de carrefours. Toutes ces infrastructures constituent des espaces linéaires ; les plantations y jouent essentiellement un rôle d'accompagnement mais aussi de décor végétal apaisant.

13 Encore aujourd'hui, la forme la plus répandue de l'arbre tunisois est l'alignement, hérité de la tradition haussmannienne et de la période coloniale, tel qu'esquissé dans la cartographie (voir ci-dessous) réalisée lors de la thèse de doctorat. 
Figure 2. Cartographie des plantations des voies publiques de Tunis, la présence d'arbres est signalée par un code de couleur

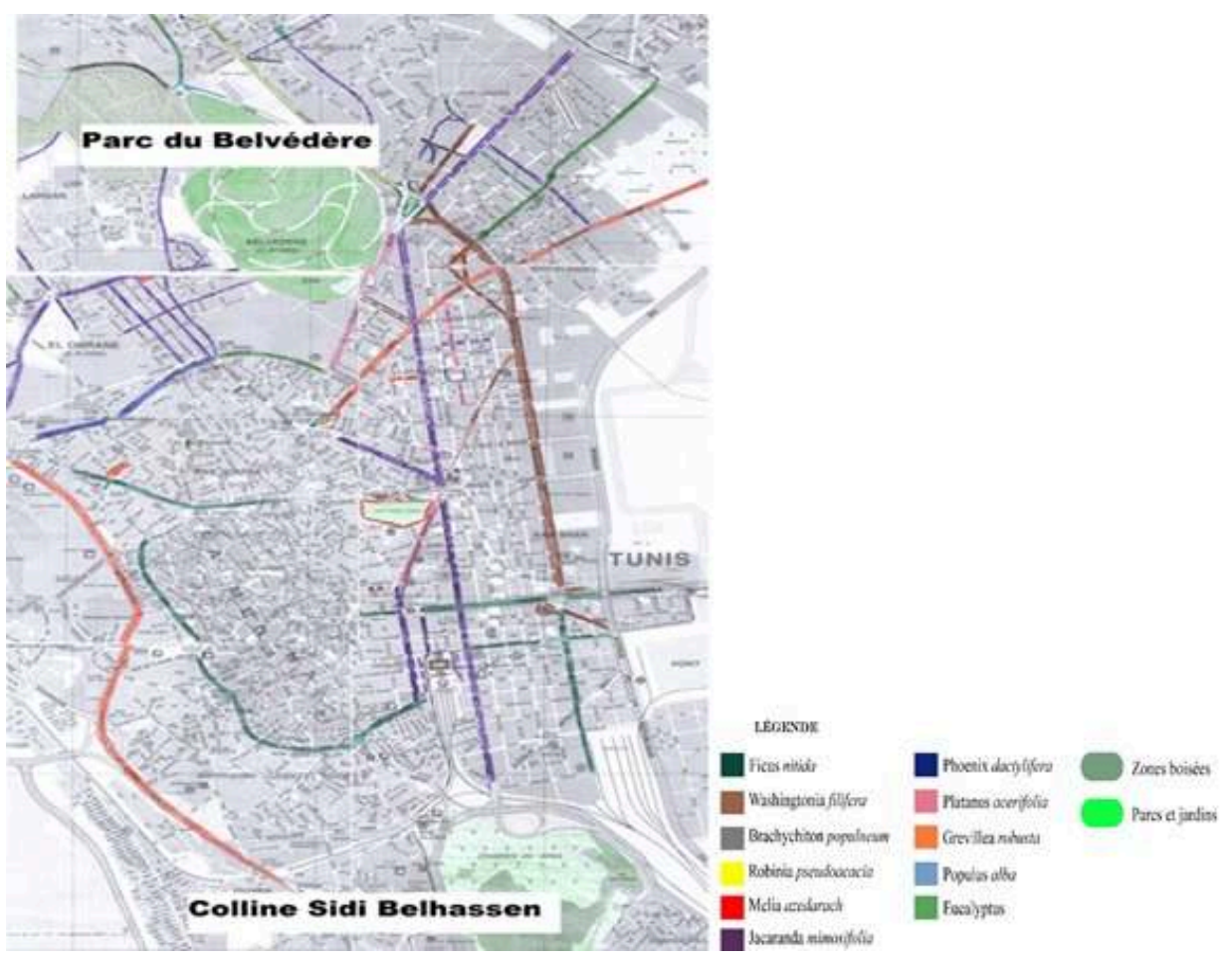

Source : Myriam Bennour, 2014

De cette structure linéaire, parle également un des responsables du service des Espaces verts de Tunis :

«Pour notre service, l'arbre a une place très importante dans la ville. On a un patrimoine assez important d'arbres d'alignement, surtout de la période coloniale. D'ailleurs, après cette période, nous n'avons pas beaucoup évolué en termes de plantation et de choix d'espèces [...]. Ensuite, avec les nouveaux quartiers, on a essayé de développer ce thème, qui est très important, parce que, généralement, quand on parle arbre, on veut dire le plus souvent arbre d'alignement.» (Un responsable du service des Espaces verts de Tunis (Agba), 2011.) 
Figure 3. Rue du Docteur-Conseil à la Cité-jardins (période coloniale)

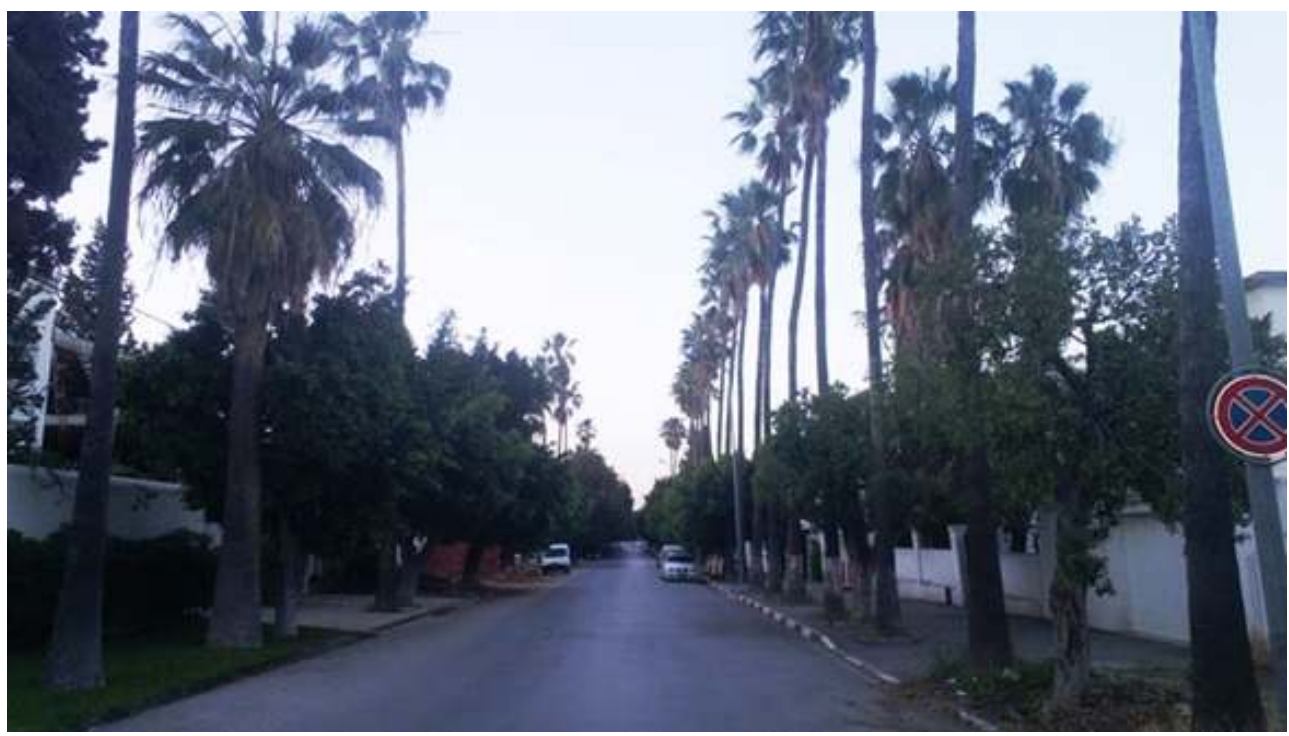

La voie est plantée en alignement mixte : Ficus nitida et Washingtonia filifera.

Source : Myriam Bennour, 2013.

Longeant les boulevards, les avenues et même les rues plus modestes, ces rangées d'arbres (Phoenix canariensis, Ficus nitida, Jacaranda mimosifolia, notamment) marquent l'espace public, le délimitent en fonction des usages (piétons, automobiles) et dirigent ainsi les perspectives (Bennour, 2014). À ce propos, on peut remarquer, dans la figure 2, les alignements de l'avenue Mohamed-V (en violet et en marron) dirigeant la perspective d'un côté vers le parc du Belvédère et de l'autre vers la colline de SidiBelhassan.

16 Le choix se porte souvent sur des espèces à feuillage persistant, notamment pour la forme taillée en rideau (c'est le cas des Ficus nitida). Ici, la fonction principale de l'arbre est surtout utilitaire. Les services techniques utilisent les arbres en rideau partout où le besoin de protection se fait sentir et pour des fins diverses réelles ou hypothétiques (écran visuel, antibruit, ombre...). D'autres espèces sont choisies pour des raisons différentes, comme une floraison qui contraste avec les couleurs de la ville. C'est le cas pour le centre historique, comme nous l'a expliqué le directeur de l'Embellissement de la ville de Tunis :

«L'une des choses sur lesquelles ont travaillé les colons, c'est l'utilisation pour les alignements d'un certain type d'arbres que l'on ne retrouve pas ailleurs que dans ces quartiers (anciens quartiers phares de la ville européenne). Citons l'exemple de la combinaison entre Grevillea et Jacaranda dans l'avenue Charles-Nicole de la Cité olympique (à côté du stade), parce que c'était à l'époque une allée qui conduisait au parc du Belvédère. »

Ainsi, le type d'espèce plantée devient un élément structurant de la ville, un moyen permettant de s'y repérer. À Tunis, l'avenue Mohamed-V est vite reconnue grâce à son alignement de Phoenix canariensis que l'on repère de loin et que l'on identifie même avant les bâtiments. Ou encore, l'exemple de l'avenue Alain-Savary (au nord du gouvernorat de Tunis), dont le nom est même parfois occulté au profit de celui de ses alignements de Jacaranda mimosifolia. En effet, on la qualifie volontiers de « rue aux arbres à fleurs violettes ». 


\section{L'échelle du parc et du jardin : en isolé ou en bosquet}

Autre héritage de la tradition haussmannienne, les places et jardins plantés d'arbres abondent encore aujourd'hui dans la ville de Tunis (figure 4). Ces nids de verdure au cœur de la ville fractionnent l'espace bâti et créent des fenêtres visuelles vers le tissu bâti.

Figure 4. Carte de localisation des espaces verts du gouvernorat de Tunis

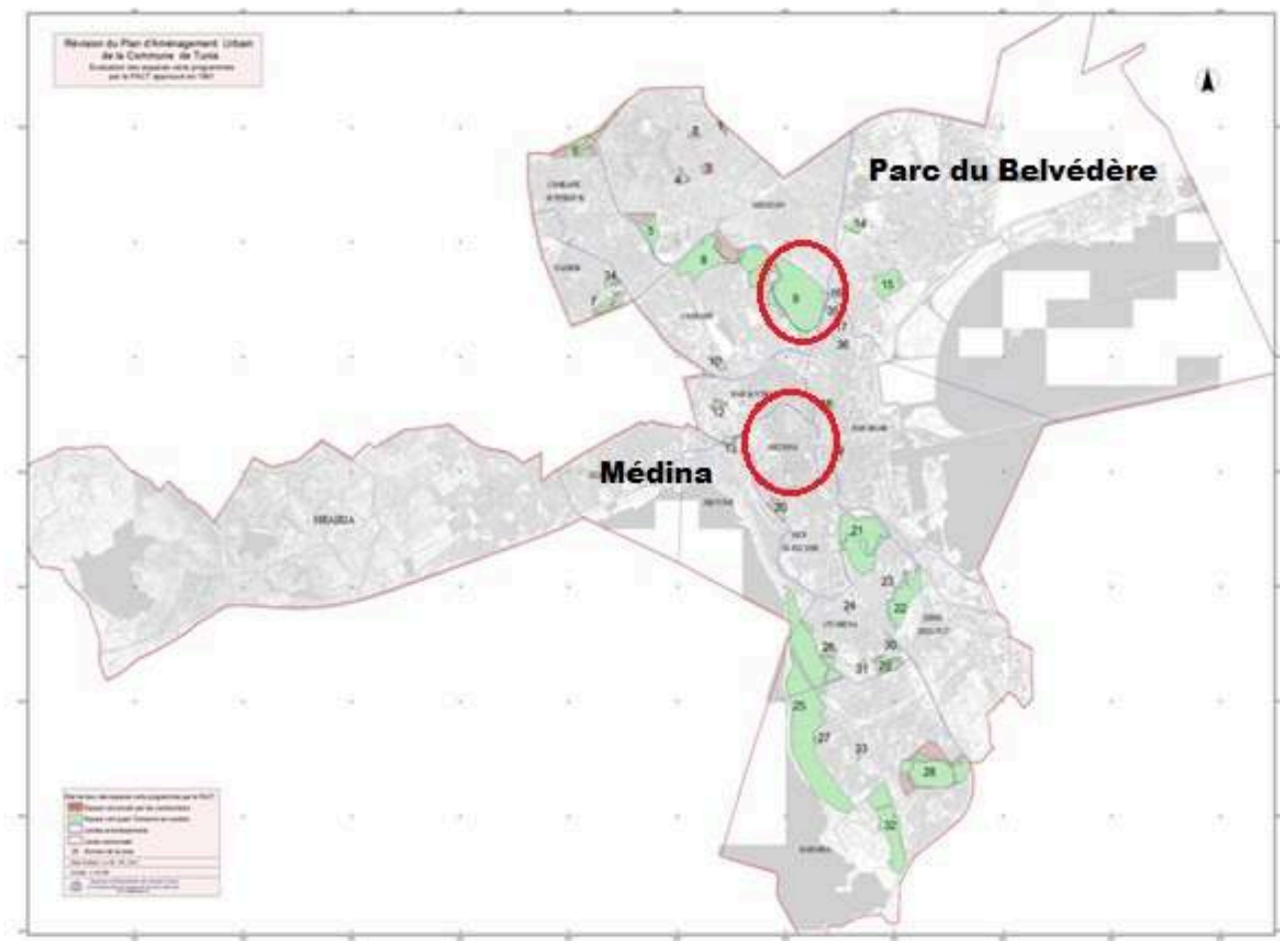

Signalés en vert, ces espaces sont particulièrement présents dans le centre historique et dans la zone résidentielle au nord.

Source : municipalité de Tunis, révision du plan d'aménagement urbain de la commune de Tunis. le constituant essentiel de l'organisation d'un parc, d'un square ou d'une place afin d'y créer un espace de détente au cœur de la ville. La qualification de marqueur de l'espace urbain est fréquemment utilisée pour les arbres situés sur les places. En effet, ces derniers sont un atout incontestable pour l'attractivité de la ville. Ils permettent ainsi de créer des lieux extérieurs de vie publique, des sortes de «bulles» pour les usagers qui en ont besoin. À cette échelle, les arbres viennent marquer le territoire de la place en signifiant une rupture avec la ville inconfortable et bruyante. De fait, ils marquent la composition urbaine, soulignent des contrastes avec le bâti, caractérisent des lieux clés par la couleur du feuillage, dressent des plans intermédiaires dans l'espace ou encore donnent du relief à des surfaces trop uniformes.

L'arbre en isolé a d'autres fonctions à son actif. En effet, d'un plus haut intérêt décoratif en principe, il est, par exemple, utilisé en contraste plastique avec un fond uniforme, qu'il soit minéral ou végétal. Détaché en avant des massifs, il sert ainsi au marquage et à la signalisation des lieux. Doté d'un port pittoresque, il attire les regards. Ainsi, il est 
constitué en point d'appel visuel. Dans ce type de plantation, l'arbre peut avoir sa propre signification et possède souvent une dimension symbolique.

\section{L'arbre en tant que porteur de mémoire}

Quand on parle de l'arbre, on doit tenir compte de deux facteurs. En effet, il y a l'espèce, représentée par un nom, une apparence commune et un patrimoine génétique, qui désigne une population d'individus qui en principe peuvent se reproduire entre eux. C'est une notion scientifique. Et puis, il y a l'individu arbre qui de fait ne ressemble pas exactement à un autre individu de la même espèce. Il est à la fois identique (il fait partie de la même espèce) et distinct des autres.

Immatérielle, l'espèce n'est pas une chose palpable. Elle est exprimée par des indicateurs anatomiques et génétiques, des représentations objectives, par exemple les couleurs de fleurs et la taille des feuilles. Même en l'absence de spécimens plantés, l'espèce peut continuer à exister (par les mots), voire même à prendre de l'importance dans le monde. S'agissant de bien naturel, c'est alors la survie de l'espèce qui prime. Dans les références mythiques et religieuses concernant les arbres, c'est d'ailleurs à l'espèce tout entière que sont attribuées les valeurs : par exemple l'olivier est symbole de paix (pensons à l'olivier de la mythologie, offert par Athéna à la ville d'Athènes en symbole de paix et grâce à qui elle est devenue la protectrice de la ville qui prit son nom) (Grimal, 1999) et le figuier de fertilité. Ainsi, dans ce cas, ce n'est pas un objet, un bien en particulier que l'on doit protéger, mais toute l'espèce.

Or, la ville est un milieu totalement artificialisé. Les processus de mise en paysage sont pour la plupart, sinon tous, directement liés au citadin et au regard qu'il porte sur ce qui l'entoure. S'agissant de l'arbre, il est naturel par essence, technique par nécessité mais, en milieu urbain, il devient avant tout un produit culturel. À ce titre, un seul individu arbre peut se poser en témoin de l'histoire locale tout autant qu'un vieil édifice. Si le spécimen est d'un âge avancé, il aura vu naître, s'épanouir puis disparaître d'anciennes civilisations ou cultures, et éveillera ainsi, chez ceux qui en connaissent l'existence, un vif intérêt qui les poussera à le préserver lui seul, indépendamment d'autres représentants plus jeunes de la même espèce. La dignité de ce vieil arbre vénérable entraînera son respect par tous sans nécessairement aboutir à sa perpétuation. Il n'est patrimoine ou plutôt bien public que le temps de sa fin de vie.

Tunis possède quelques spécimens notables d'arbres (par leur âge ou par leur port), dont les plus impressionnants sont des Ficus macrophylla situés dans la ville européenne. Le premier, situé au parc du Belvédère, appelé aussi « l'arbre de Tarzan » est devenu un monument du fait de son port impressionnant, de ses branches tortueuses et volumineuses devenues une attraction pour plusieurs générations de visiteurs du parc, si bien qu'il a été très souvent cité lors des entretiens réalisés avec la population, de même qu'avec les acteurs publics de l'arbre (les responsables des services des Espaces verts de la ville de Tunis, les membres de l'Association des Amis du Belvédère (AAB) qui a notamment organisé un événement autour de sa valorisation le 9 novembre 2014 lors de la fête de l'Arbre, célébrant par la même occasion les 125 ans de ce Ficus). 
Figure 5. Ficus macrophylla du parc du Belvédère, « l'arbre de Tarzan »

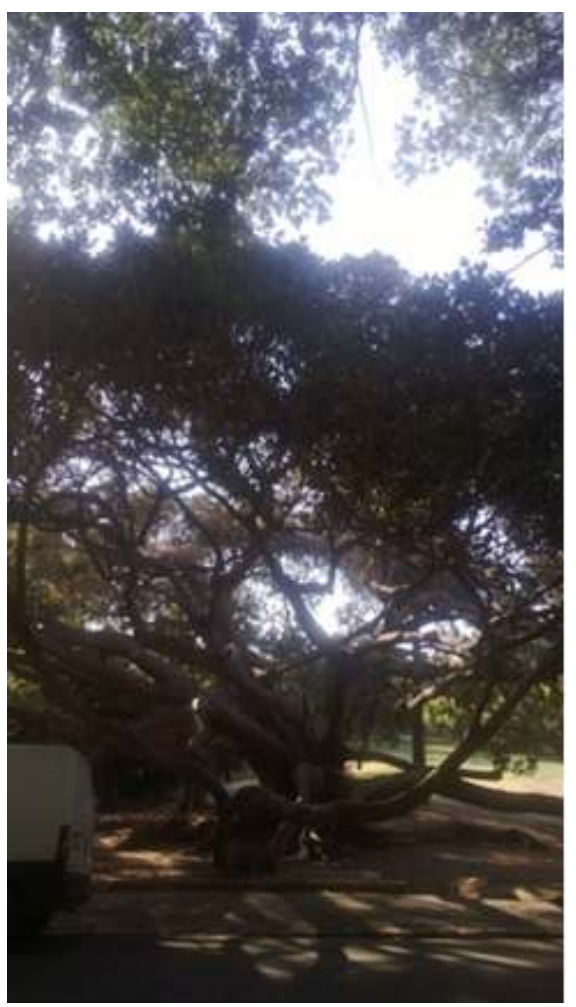

Source : Myriam Bennour, 2013

Le deuxième exemple concerne les vieux ficus de la place de la Monnaie. Située dans la zone de transition entre ville arabe et ville européenne, cette place est au cœur d'une zone marchande " anarchique ». Bien que peu mise en valeur, elle a suscité l'intérêt de la société civile. Celle-ci, poussée par l'Association des Amis du Belvédère, s'est opposée au projet de la municipalité de Tunis qui visait sa transformation en parking au début des années 1990 (et de ce fait prévoyait l'abattage des arbres). Notons qu'aujourd'hui, c'est la municipalité elle-même qui défend la conservation et la mise en valeur de cette place et de ses arbres bicentenaires. En effet, un projet porté par la municipalité de Tunis, avec le soutien de l'office du tourisme, a été programmé en vue d'une refonte totale de ce lieu, incluant une structure en béton armé pour protéger les racines des Ficus macrophylla, aujourd'hui apparentes, et la transformation des voies de circulation immédiate en circuit piéton (pour décongestionner la place). Il faut noter néanmoins que, bien que ce projet ait été approuvé par les autorités concernées et que le financement ait été versé, les travaux n'ont pas pu être lancés par souci sécuritaire (les travaux devaient commencer fin 2010, coïncidant avec le début des manifestations populaires qui ont eu lieu en Tunisie). Cela démontre bien que le désir de préservation, voire de mise en patrimoine, naît d'un élan spontané des citadins pour ensuite concerner les pouvoirs publics.

Il y a eu aussi la pétition, portée par l'Association des Amis du Belvédère, contre le plan d'aménagement de l'avenue Habib-Bourguiba (1999) qui visait à donner un nouveau visage à l'avenue (Ben Becher, 2003), mais impliquait l'abattage des alignements de Ficus nitida. Cette pétition a été signée par plus de 200 personnalités (des acteurs, des personnages publics, des membres de l'AAB, certains fonctionnaires de la municipalité de Tunis), des citadins, et elle a été publiée dans l'hebdomadaire Réalités. Elle a été le 
point de départ d'une refonte du projet à partir de l'année 2000 dans un souci de compromis, selon un fonctionnaire de la municipalité de Tunis, architecte de formation, ayant pris part au projet :

«Les gens étaient contre. Ils ont refusé le projet de peur que les arbres ne puissent survivre. On a d'ailleurs dû louer une machine de l'étranger pour pouvoir les déplacer avec leur terre jusqu'au Jardin méditerranéen. Le projet n'aurait pas pu se faire sinon. [...] Il faut dire que ces ficus représentent un patrimoine. Avec les oiseaux (des étourneaux) qui viennent y nicher, ils constituent le cachet de l'avenue. »

Certaines des raisons qui permettent d'expliquer de tels élans de protection envers les arbres de l'espace urbain sont propres à la société tunisienne. En effet, en Tunisie, comme dans d'autres pays anciennement colonisés, ce n'est qu'avec le temps, permettant une certaine distanciation par rapport aux réalisations coloniales (comme dans le cas des Ficus nitida de l'avenue Bourguiba, mais aussi des Ficus macrophylla bicentenaires), que le processus d'appropriation symbolique des arbres coloniaux peut commencer. Concernant les années 1990 et 2000, nous parlons d'une génération d'hommes et de femmes qui n'ont connu la colonisation que dans l'enfance ou à travers des souvenirs parentaux. Ils ont été de fait capables de s'approprier une part d'un passé qui s'éloigne (Matri, 2008).

Plus près de nous, en 2016, une vague de protestation s'est élevée contre le propriétaire d'un restaurant situé dans la banlieue nord de Tunis, qui avait fait abattre un eucalyptus de la voie publique, celui-ci obstruant la vue sur son commerce. Ici aussi, il s'agissait d'une plantation coloniale. Mais pour les signataires de la pétition, c'était avant tout une partie de l'histoire commune, un élément de la mémoire du quartier qui avait été détruit.

31 Il est à déplorer qu'à Tunis - en dehors du projet présenté ci-dessus - il n'existe pas à ce jour de mesures de reconnaissance, de protection, voire de mise en valeur des arbres de la part des pouvoirs publics, exception faite de l'olivier. Cependant, le fait que ces arbres, garants de la mémoire collective, existent toujours, grâce à la société civile qui s'interpose lorsqu'ils sont en danger, apparait comme une preuve de l'intérêt qui leur est porté, de même que du sens symbolique qui les accompagne.

\section{L'arbre symbole spirituel}

32 La population tunisoise est encore aujourd'hui profondément attachée à ses traditions spirituelles et à ses croyances. Ainsi, elle tend à respecter les préceptes religieux au quotidien (Abdelkafi, 1989). Dans le Coran, ainsi que dans les autres dogmes monothéistes, nombreuses sont les références aux arbres. Certains y sont cités comme sacrés, d'autres comme bénéfiques ou encore maléfiques. Le terme chajar (achjâr au pluriel) désigne, dans le vocabulaire coranique, aussi bien les arbres proprement dits que tout végétal, et plus précisément ce qui pousse avec une tige, volumineuse ou fine. Dans le Coran certains arbres sont nommés, comme l'olivier, le palmier dattier, le grenadier, l'acacia et le figuier, mais d'autres, comme l'arbre du paradis, n'y sont pas identifiés avec précision. Ce n'est donc pas un hasard de découvrir que les Tunisois accordent aux arbres cités dans les textes une place de choix, comme en témoigne l'entretien avec une Tunisoise de 69 ans au sujet d'un palmier planté sur le trottoir en face de chez elle, et qui gênait la clôture : «Que voulez-vous qu'on y fasse ? On ne peut pas l'abattre, c'est un palmier, ce serait hram! (mot en arabe signifiant interdit par la 
religion). » En effet, le palmier jouit d'un statut particulier parmi tous les arbres. Selon le Coran, il s'agit de l'arbre qui ressemble le plus à l'homme; il aurait été créé de la même argile qu'Adam. D'après Ibn Omar (compagnon de Mahomet), « le Prophète a dit : "Il existe un arbre d'entre les arbres dont les feuilles ne tombent pas et qui est l'emblème du Musulman. [...]" On demanda à l'Envoyé de Dieu à quel arbre il faisait allusion. Il répondit: "Au palmier".» (Al-Boukhari, 2007.) Ces essences citées dans le Coran se retrouvent dans de nombreux entretiens avec les Tunisois lorsqu'ils décrivent les plantations de leur jardin, ou énumèrent les espèces qu'ils voudraient voir davantage dans la ville et dans les parcs (Bennour, 2014).

Enfin, on ne peut parler des arbres en tant que symbole religieux sans évoquer l'olivier. Il s'agit de l'une des rares espèces arborées à jouir d'une protection de la part des services des Espaces verts, puisque leur abattage est interdit par la loi (loi $\mathrm{n}^{\circ}$ 2001-119 du 6 décembre 2001, modifiant la loi n 61-20 du 31 mai 1961, portant interdiction de l'abattage et de l'arrachage des oliviers). C'est le cas notamment de cette ancienne oliveraie (figure 6), enclavée dans le tissu urbain. Les services de la municipalité n'ont eu d'autre choix que de l'intégrer à la ville en la transformant en aire de jeux.

Figure 6. Une ancienne oliveraie réhabilitée par les services publics urbains en aire de jeux dans le quartier d'Ibn Khaldoun

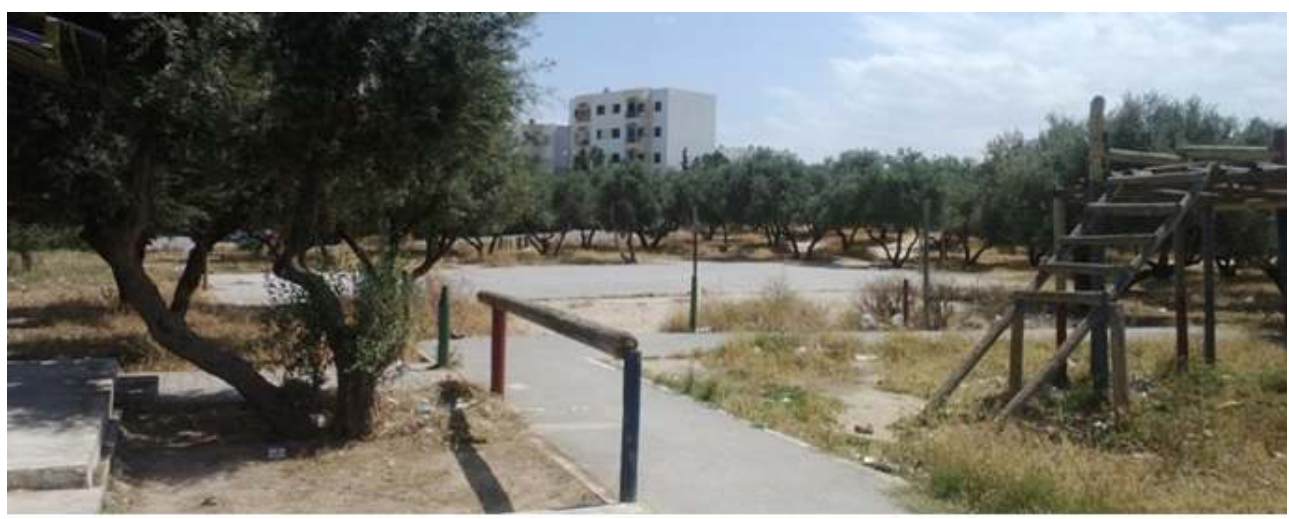

Source : Myriam Bennour, 2012

\section{Le cas particulier de la médina et les arbres}

Le cas particulier de la médina de Tunis mérite qu'on s'y arrête, en ce sens que c'est une ville minérale par essence et que de tout temps, les arbres y ont été exclus (du moins leur présence matérielle).

La médina de Tunis est un espace historique protégé qui a bénéficié d'un regain d'attention depuis les années 1970. Il s'est ensuivi le classement de la médina de Tunis dans le patrimoine mondial de l'Unesco. Depuis cette période, les études et les projets de réhabilitation ne cessent de voir le jour. La médina est certes un patrimoine historique, mais aussi un lieu de vie pour plus de cent mille habitants. Dans ce sens, les projets de restauration doivent prendre en considération autant les caractéristiques du lieu historique que les besoins des usagers.

La ville arabe fondée à la fin du viI siècle s'est formée autour de la mosquée Zitouna (de l'olivier). L'unité de base de la médina est le bâtiment à cour (patio), utilisé indifféremment pour les maisons et les équipements publics qui, assemblés à d'autres 
bâtiments de même type, forment des îlots desservis par un réseau viaire hiérarchisé. La largeur des rues n'est pas uniforme. La perspective est discontinue; on découvre le paysage urbain séquence par séquence, en avançant dans les rues.

À l'emplacement de la Kasbah, une forteresse édifiée dans la première moitié du XIII ${ }^{\mathrm{e}}$ siècle, on retrouve aujourd'hui les principaux ministères et l'hôtel de ville de Tunis. Avec le temps, le tissu urbain s'est densifié au maximum, souvent au détriment des anciens jardins (où l'on retrouvait essentiellement agrumes, grenadiers, pruniers et oliviers). Traditionnellement, l'arbre a donc une place limitée dans la médina.

Aujourd'hui dans le cadre de la réhabilitation du patrimoine, quelle place est accordée à l'arbre ? Est-ce qu'on ne contredit pas l'esprit des lieux en essayant de l'y intégrer? En regardant de plus près les noms des portes et des rues qui composent la médina, on constate que, bien que l'espace ne leur laisse pas de place, l'esprit des arbres est quant à lui, bien présent. Ainsi, on retrouve Bab El Khadra (littéralement porte du Vert) qui, à l'époque, donnait accès aux champs de cultures maraîchères et arbustives. De même que Bab El Alouj qui s'est d'abord appelé Bab Erehiba (signifiant porte de la PetiteEsplanade) du fait qu'elle reliait le palais de la Kasbah aux parcs royaux de Ras-Tabia et d'Abou-Fîhr.

Pour ce qui est des noms des rues, certaines s'appellent rue du Palmier, du Grenadier, ou encore rue des Figues et rue des Prunes. Il y a deux explications qui sont les plus probables et d'ailleurs les plus connues. La première est que la ville, au cours de son extension, a empiété sur des vergers et autres jardins qui contenaient ces essences. La seconde est que, durant les périodes de dépeuplement ou de ruine, certaines espèces ont pu réapparaître (entretien avec Jamila Binous, historienne spécialiste de la médina de Tunis). Cela montre que, bien que peu présent dans la ville historique, l'arbre y a tout de même laissé sa marque.

Tout au long de leur histoire, les arbres ont été synonymes de villégiature et de luxe pour les Tunisois. Car ils étaient localisés dans les grandes demeures de la médina, ainsi que dans les palais et les résidences de villégiature des aristocrates. Aujourd'hui, avec l'évolution de l'espace urbain de la médina due aux nouveaux arrivants et aux nécessités de la vie moderne, l'arbre fait de plus en plus son entrée dans ses rues. On a néanmoins remarqué que les aménagements paysagers étaient concentrés autour de la place du Gouvernement (siège des principaux ministères) ainsi que dans le circuit touristique restauré au centre de la médina. 
Figure 7. Localisation dans la médina de la zone restaurée

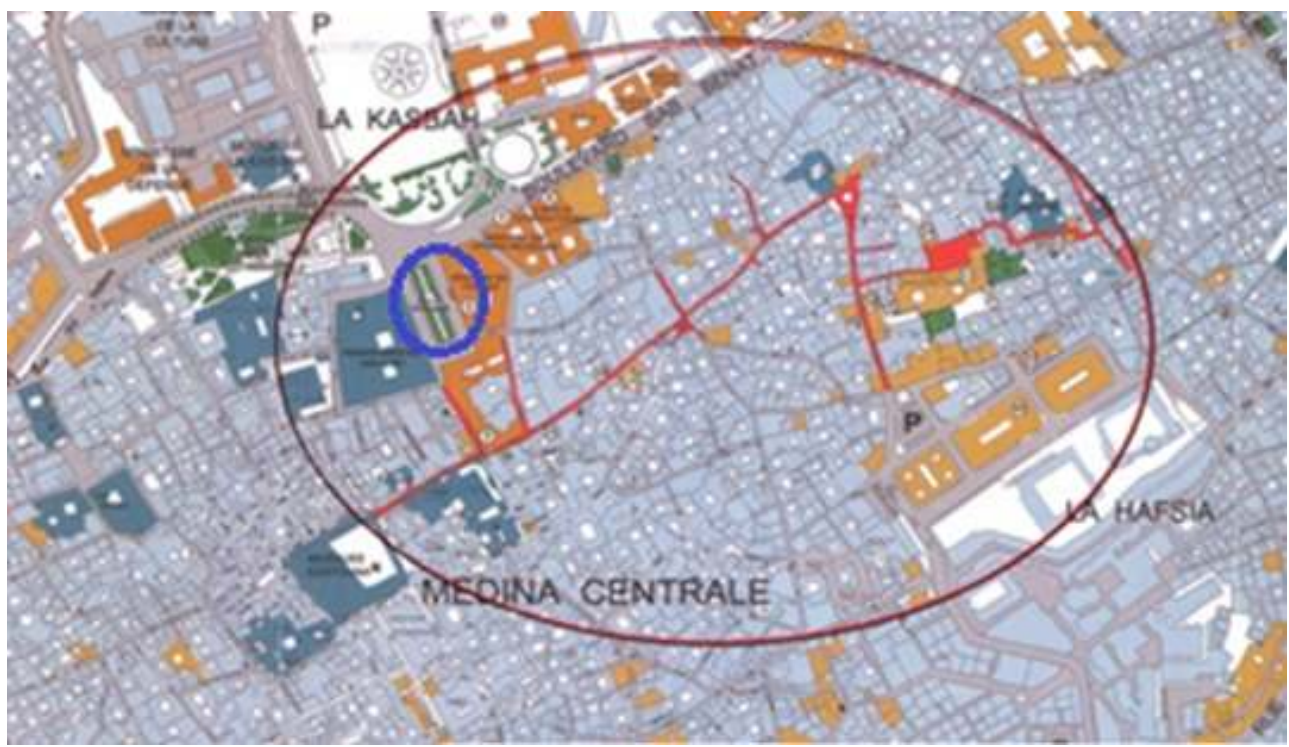

La rue du Pacha-Hafsia (encerclée en rouge), et la place du Gouvernement (encerclée en bleu).

Source : Plan de l'Association de sauvegarde de la Médina, 2012.

Ces changements restent néanmoins concentrés sur les axes principaux (de la rue du Pacha vers la Kasbah, en passant par la place du Tribunal), correspondant aux circuits touristiques et aux adresses prestigieuses (le palais Kheireddine, le Diwan (espace d'exposition et de vente de l'artisanat), le restaurant Dar El Jeld, le club Tahar Haddad, l'espace culturel Bir Lahjar...).

42 Jusqu'à aujourd'hui, l'Association de sauvegarde de la Médina (ASM) demeure l'acteur principal de l'aménagement de l'espace public de la Médina. Elle a ainsi entrepris un bon nombre de projets (les plus connus étant ceux effectués à Bab Souika, Halfaouine, ainsi que ceux de la rue du Pacha-Hafsia-Kasbah illustré dans la figure 7). 
Figure 8. Place du Tribunal en face du palais Kheireddine, plantée de Washingtonia filifera, récemment aménagée

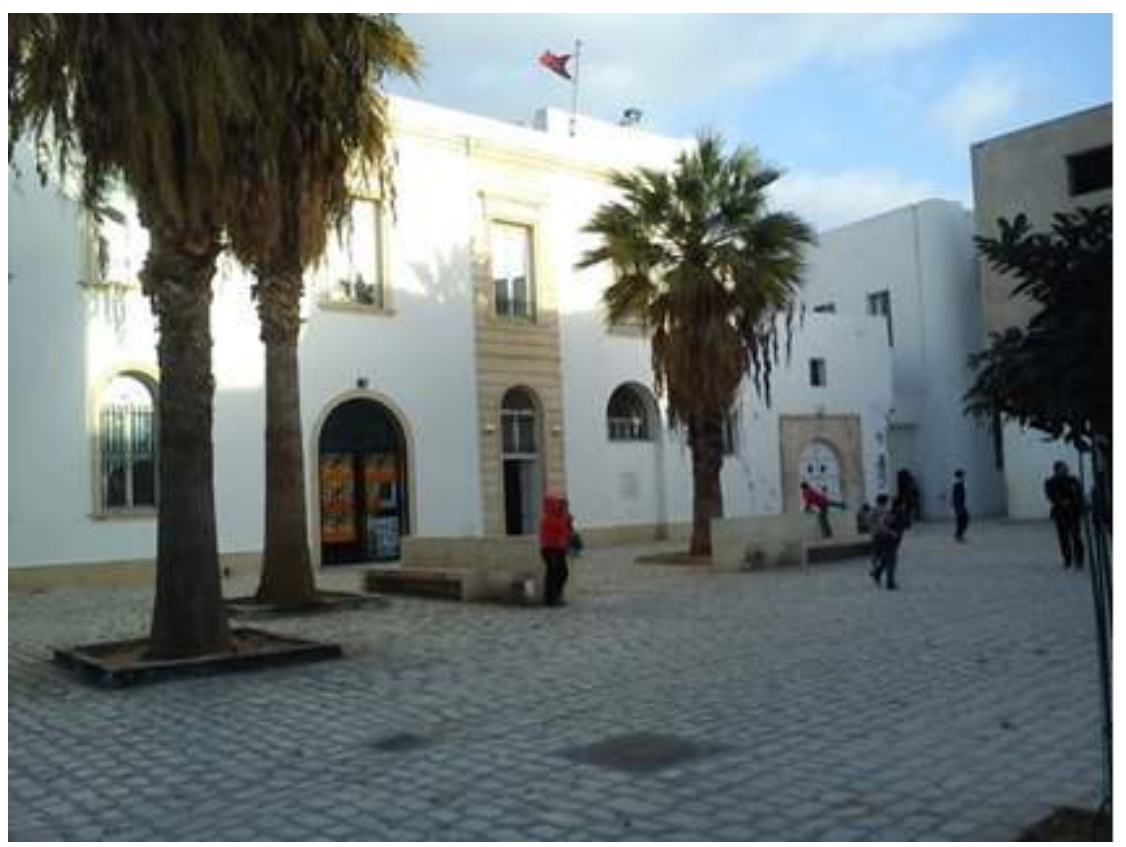

Source : Myriam Bennour, 2012 habitants et les priorités actuelles. Elle a donc consacré une part de ces réalisations à l'amélioration du cadre de vie des habitants. Ceci s'est exprimé sur le terrain par l'aménagement de diverses places et placettes (Bir Lahjar, Halfaouine, Bab Souika), le plus souvent accompagnées de plantations - pour la plupart des Phoenix (canariensis et dactylifera) ou Washingtonia filifera, des agrumes et des oliviers -, à savoir des espèces en accord avec les valeurs symboliques que nous évoquions plus haut. Ces lieux ont été réalisés sur des espaces vacants pour certains, à la place d'anciennes constructions (insalubres qui ont été détruites comme à Bab Souika) pour d'autres. Mais ce qui permet à ces projets de perdurer et de ce fait à la Médina de vivre une seconde jeunesse, ce sont bien les intellectuels et artistes de toutes natures qui, attirés par son nouveau décor, y organisent des manifestations (projections de films, clubs, expositions d'art, circuits de découverte...) ou alors qui ont décidé de s'y réinstaller comme l'ancien directeur du théâtre municipal Driss Adel.

C'est donc, en partie, par la réintroduction de l'arbre porteur de sens dans la médina que celle-ci a pu gagner en attractivité et correspondre mieux au goût de ses habitants et visiteurs actuels.

\section{Conclusion}

L'arbre est un élément structurant essentiel de l'espace de vie dans la ville de Tunis. Trois rôles peuvent lui être attribués. En tant que décor urbain, il prend différentes formes en fonction de son lieu d'implantation (voirie et parc). En tant que porteur de la mémoire collective, il est défendu par les habitants qui se sont mobilisés plus d'une fois lorsque des spécimens anciens d'arbres étaient menacés. Enfin, l'arbre urbain apparaît comme un symbole spirituel puisque nombre d'espèces plantées dont l'olivier et le 
palmier jouissent d'un statut privilégié dans la tradition musulmane. Ce sont d'ailleurs les valeurs esthétiques et symboliques portées par ces essences qui ont permis leur intégration au paysage de la médina.

Ainsi, de multiples valeurs esthétiques, éthiques et spirituelles sont portées par l'arbre urbain de la ville de Tunis. Réunissant fonctionnalités écologiques, décoratives, patrimoniales et croyances religieuses, il est porteur de sens pour les Tunisois. Il fabrique ainsi l'identité et la singularité sociales et culturelles des lieux urbains. À ce titre, il pourrait devenir, mieux qu'aujourd'hui, un outil incontournable tant pour l'aménageur que pour les pouvoirs publics dans la recherche de l'amélioration du cadre de vie des Tunisois, un outil à mobiliser non seulement pour les usages qu'il permet mais aussi pour les symboles qu'il porte.

Je tiens à remercier Pierre Donadieu pour ses précieux conseils.

\section{BIBLIOGRAPHIE}

Abdelkafi, J., La Médina de Tunis : espace historique, Paris, Presse du CNRS, 1989.

Al-Boukhari, Sahih al-Boukhari, T. 3, hadiths 3776-5474, Paris, Maison d'Ennour, 2007.

André, E., Traité général de la composition des parcs et jardins, Paris, G. Masson Éditeur, 1879.

Audrerie, D., Questions sur le patrimoine, Bordeaux, Éditions Confluences, Coll. « Stèles », 2003.

Babelon, J.-P. et Chastel, A., La Notion de patrimoine (1980), Paris, Liana Levi, coll. « Opinion art », 1994.

Ben Becher, F., Tunis : histoire d'une avenue, Tunis, Éditions Nirvana, 2003.

Bennour Azooz, M, « La patrimonialisation des arbres urbains, cas de la ville de Tunis ", thèse de doctorat, AgroParistech/ENSP (Versailles) et Institut supérieur d'Agronomie (université de Sousse), 2014.

Bennour Azooz, M, « Les arbres de Tunis peuvent-ils devenir un patrimoine urbain ? Pour qui ? Pourquoi ? ", Projets de paysage, 4 janvier 2012, URL : http://www.projetsdepaysage.fr/fr/ les_arbres_de_tunis_peuvent_ils_devenir_un_patrimoine_urbain_pour_qui_pourquoi_.

Bennour Azooz, M., Donadieu, P. et Bettaîeb, T., « L'arbre à Tunis : hypothèses pour une histoire de l'espace public », Projets de paysage, 13 juillet 2012, URL : http://www.projetsdepaysage.fr/fr/ 1_arbre_tunis_hypoth_ses_pour_une_histoire_de_1_espace_public.

Grimal Pierre, Dictionnaire de la mythologie grecque et romaine, Paris, Presses universitaires de France, 1999.

Lévy, Jacques et Lussault, Michel, Dictionnaire de la géographie et de l'espace des sociétés, Paris, Belin, 2003.

Lissan al-Arab, Dictionnaire de la langue arabe, 20 vol. , Le Caire, 1879-1888 (1299-1309 Hégire).

Matri, Faîza, Tunis sous le protectorat : histoire de la conservation du patrimoine architectural et urbain de la médina, Tunis, Centre de publication universitaire, 2008. 
Tollis, C., « Pour le meilleur et pour le pire ! Les arbres en ville peuvent-ils faire patrimoine? Analyse des spatialités concurrentes arbres-riverains à Grenoble ", Vertigo - la revue électronique en sciences de l'environnement, hors-série 16, juin 2013, URL : URL : http://vertigo.revues.org/ 13736, DOI : 10.4000/vertigo.13736.

Tricaud, Pierre-Marie, « Conservation et transformation du patrimoine vivant : Étude des conditions de préservation des valeurs des patrimoines évolutifs ", thèse de doctorat en aménagement de l'espace, urbanisme, 2010, Institut d'urbanisme de Paris.

\section{RÉSUMÉS}

Sur quels critères faut-il se fonder pour conserver un arbre urbain en tant que patrimoine à transmettre? Trois hypothèses seront explorées dans le cas de la ville de Tunis. La première est liée au rôle environnemental et esthétique des arbres. Utiles à la qualité de vie et à la santé urbaine, ils doivent être conservés pour le bien-être des habitants. La deuxième soutient que les arbres plantés dans les villes peuvent être considérés comme des monuments au sens où ils évoquent la mémoire urbaine. La dernière relève de l'attachement intime, souvent symbolique, voire religieux, que les habitants peuvent éprouver pour tel ou tel arbre. L'arbre contribue ainsi à forger l'identité des citadins en marquant leur espace de vie.

What criteria should be used for preserving an urban tree as a cultural heritage to be transmitted? Three hypotheses are examined in the case of the city of Tunis. The first relates to the environmental and aesthetic role played by trees, their useful contribution to the quality of life and health in the urban environment, and the need to preserve them to ensure the wellbeing of the population. The second hypothesis supports the idea that trees may be considered as monuments in the sense of evoking an urban memory. The third hypothesis concerns a personal, often symbolic or even religious attachment of the inhabitants to certain trees. The tree thus contributes to forging the identity of the inhabitants of the city by marking their living environment.

\section{INDEX}

Mots-clés : arbres urbains, patrimonialisation, valeur symbolique, tradition musulmane, Tunis Keywords : urban trees, heritagisation, symbolic value, Muslim tradition, Tunis

\section{AUTEUR}

\section{MYRIAM BENNOUR AZOOZ}

Myriam Bennour Azooz est docteur en paysage, territoire et patrimoine de l'Institut supérieur d'agronomie de Chott-Mariem (université de Sousse, Tunisie) en cotutelle avec AgroParistech, ENSP Versailles.

myriambennour[at]yahoo[dot]com 